\title{
How to Size an Exoplanet? A Model Approach for Visualization
}

\section{Akos Kereszturi*}

Research Center for Astronomy and Earth Sciences, Hungary

\begin{abstract}
The realization and experiences with a physical exoplanet model in the education and outreach are described. During the tests with students in the classroom and adults at public demonstrations, the following conclusions were drawn: in the visualization it is effective to connect and install together the models of planets inside and outside the Solar System, where hot Jupiter's easily fit into the orbit of Mercury. The size of planets and orbital distance could be more effectively visualized with this method, using the Solar System as a context. The exoplanet model helps to expand the imagination of the audience on how does a planetary system look like, what is getting important as diverse views of explanatory systems emerging in recent years. This inexpensive model is useful in the education and outreach above all to make the audience more familiar with the parameters of exoplanets (here sizes, distances and partly masses), and it gives new input also for those persons who regularly read papers and news on exoplanets.
\end{abstract}

Keywords: Solar system; Mercury; Jupiter; Exoplanets; Planet; Astrobiology

\section{Introduction}

The aim of this work is to present a method and experiences using it, to visualize exoplanets in the classroom and in a summer camp in order to popularize astrobiology. Below we describe the basic idea behind the visualization, some important details of the realization, and our experiences to draw a conclusion on the affectivity of such method. This project is the continuation of our work in the education of astrobiology related topics both in the secondary schools and at university level in Hungary [1-4]. Several publications appeared in the last years on how exoplanet could be used in the education [5] including the use of online accessible telescopes [6] and simulations [7] with the description of classroom activities and teaching standards [8].

Being an important and also popular subtopic of astrobiology, exoplanets often appear in the popularization related issues [9-11] and in the education. Although this hot topic is in the focus, only images were used to visualize the size of these bodies, and the methods often lacks the visualization of distances proportional to the real stellar and planet sizes. Here we describe a simple homemade plastic model of exoplanets that was tested with students and adult audience both as part of the education and as a public outreach activity.

The tests of the model were realized by the help of the Hungarian Astronomical Association and the Karoly Nagy Astronomical Foundation, as these organizations have good connections with secondary schools in Hungary and give regular astronomy related lectures there. We were interested in the possibility of visualizing basic parameters of exoplanets, like their diameter, distance and mass, especially relative to the same parameters of the planets known in the Solar System. The comparison to Solar System object put this discussion into known context, as the students had already learned about the Solar System.

\section{Materials and Methods}

During this work we planned, developed and produced models for the planets in the Solar System, model for the Sun (that served as model for central stars of exoplanets too) and models also for the selected exoplanets. We taught the students in the classroom course (kept at the observatory) and in a summer astronomy camp. We also held public demonstrations for adult persons from the public as popularization lectures. The visualization methods were tested at five times (three classroom sessions, and two sessions in astronomy camps) with different groups and persons, altogether for 178 participants at these lectures. 132 of them were secondary school students with interest in astronomy; the rest was composed of adults with interest but without a degree in astronomy. There were no contact outside the presentations between the lecturer and the audience, so the result of the survey is representative and excludes any personal motivation that could influence the result. The presentation of the model was accompanied by an oral lecture including images projected by a beamer on the basic characteristics of exoplanets that have been observed up to that date.

We used simple anonym written tests to survey what the audience understood from the presentations, and analyzed the result with statistical method. The students did not receive grade for it, so there was no reason to copy from each other. We also used personal discussions during the presentations (questions and answers from both sides) to identify the effect of the presentations beyond the written survey. Here the interpretation is somewhat subjective, but we only used those findings that looked evident from the conversations and related to substantial part of the audience and not only to few persons.

The compiled exoplanet model uses a simplification: the central star in every case was taken to be equal to a solar sized main sequence star and was visualized by a large white (in the camp) or a yellow (in the observatory) ball made of plastic. Using only one stellar model for all the exoplanet's central stars means we demonstrated all the selected exoplanets and also the Solar System planets to orbit around the same large ball. Exoplanets and Solar System planets were made of simple solid plastic balls with different size, and were painted manually by two colleagues. During the visualization all the selected exoplanets were put around the central star at the same time, e.g. exoplanets orbiting around different stars were visualized at the same time and location,

${ }^{*}$ Corresponding author: Akos Kereszturi, Research Center for Astronomy and Earth Sciences, Hungary, Tel: +36 1391 9355; Fax: +36 1 2754668; E-mail: kereszturi@konkoly.hu

Received January 16, 2013; Accepted March 20, 2013; Published March 25 2013

Citation: Kereszturi A (2013) How to Size an Exoplanet? A Model Approach for Visualization. Astrol Outreach 1: 101. doi:10.4172/2332-2519.1000101

Copyright: (c) 2013 Kereszturi A. This is an open-access article distributed under the terms of the Creative Commons Attribution License, which permits unrestricted use, distribution, and reproduction in any medium, provided the original author and source are credited. 
next to each other - while the lecturer explained these objects belong to different systems and orbit around different stars.

\section{Results}

In the Results section we describe the used models for the demonstration we developed, while the experiences gained during using them are summarized in the Discussion section. We selected the exoplanets to visualize in 2008, before the relatively large number of smaller exoplanets discovered by Kepler. Around 2008 and 2009 most of the known exoplanets were Jupiter sized with some Neptune class object, but substantially fewer than it is known during the compilation of this paper at the beginning of 2013. As a result, the objects that were visualized are representative for those objects that were known around 2008, and these were mostly hot Jupiter's [12-14]. The used data of these exoplanets, including the planets from the Solar System are listed in Table 1, where their diameters and orbital distances are indicated as real values and also those that are valid in the model. The model used the scale 1:2316666666 to make the sizes and distances compatible with the human walking distance, but this still caused some restrictions regarding where could be the model installed (see later in the 3.2. section).

\section{Characteristics of the model}

We used two different sets for the installation of the models: hanging of picture like plates on the wall at the Observatory, and putting the models on the top of sticks in the astronomy camp (at the meadow next to the lecture room). More information could be visualized when the models were fixed on a plastic plate on the wall, and this later installation is described in details below.

During the classroom activity all exoplanets were fixed at the top of an A5 sized solid plastic plate with written and graphical information below the model on a sheet of paper (Figure 1). The following parameters were indicated for each object: exoplanet's name, parameters of the central star (including id. number of the star, spectral type, constellation, distance and apparent brightness). At the bottom of the plate the exoplanet's parameters were indicated, including orbital period, average distance from the central star, mass and diameter. The latter two were indicated graphically, together with the corresponding size of Jupiter and Earth from the Solar System for comparison. In Figure 1. the parameter sheet is visible of HD $149026 \mathrm{~b}$ as an example.
For the visualization of the mass the following method was used: if the corresponding mass of the exoplanet is smaller than those of Jupiter, than the corresponding fraction of a simple Jupiter image was indicated with high contrast (as a pie diagram). In the case of exoplanets with mass larger than that of Jupiter, the corresponding number of Jupiter images was printed overlapping each other. One example is visible in Figure 2. where the 11.8 Jupiter mass of the $\mathrm{XO}-3$ $\mathrm{b}$ exoplanet is indicated. This method was found to be physically compact (no larger plates were necessary for more heavy planets) and also expressive regarding the graphical representation.

\section{Installation of the model}

We installed the models at the Polaris Observatory on the wall, starting with the solar globe (cut into half, and put this half on the wall to economize with the spaces in the room), while the plates with the exoplanets were hanged on cords, like small frame of images (Figure 3 ). The distance from the solar globe was proportional to the size of exoplanets and it represented well the small distance of hot Jupiter's from their star too We also indicated the distance of Mercury at the opposite corner of the same room using the same scale.

The indication of the other planets beyond Mercury in the Solar System was not possible in the room, as they are too far away from the central star using this scale. The hot Jupiter's and all of our planets could be installed together only outdoor in the summer astronomy camp. In the classroom of the observatory only Mercury could be installed beside the exoplanets at the same room. Beyond Mercury (from Venus to Neptune) we also put on these plastic models close to each other with a written table above them indicating this part is not to scale, and the lecturer emphasized with oral presentation that here the model does not keep the distance scale any more from here. This way in the used model the planets between Venus and Neptune are much closer to each other than in a proportional model (so the distance scale is lost), but the sizes are still proportional to each other. We also mentioned where would have been these planets located in this model if we have enough room for the installation (we mentioned distances that are familiar with the visitors, like at the end out the court, at the next street crossing the main road etc.).

For the visualization of the masses of exoplanets, at the astronomy camp we used a simple method: collected large rocks with roughly the

\begin{tabular}{|c|c|c|c|c|c|c|}
\hline Object & real diameter $(\mathrm{km})$ & model diameter $(\mathrm{km})$ & model diameter $(\mathrm{cm})$ & real solar distance $(\mathrm{km})$ & model solar distance $(\mathrm{km})$ & model solar distance $(\mathrm{m})$ \\
\hline Sun & 1390000 & 0.0006000 & 60 & & & \\
\hline Mercury & 4880 & 0.0000021 & 0.211 & 58000000 & 0.02504 & 25.04 \\
\hline Venus & 12102 & 0.0000052 & 0.522 & 108000000 & 0.04662 & 46.62 \\
\hline Earth & 12756 & 0.0000055 & 0.551 & 149600000 & 0.06458 & 64.58 \\
\hline Mars & 6792 & 0.0000029 & 0.293 & 228000000 & 0.09842 & 98.42 \\
\hline Jupiter & 142796 & 0.0000616 & 6.164 & 778000000 & 0.33583 & 335.83 \\
\hline Saturn & 120536 & 0.0000520 & 5.203 & 1428000000 & 0.61640 & 616.40 \\
\hline Uranus & 51120 & 0.0000221 & 2.207 & 2869000000 & 1.23842 & 1238.42 \\
\hline Neptune & 49520 & 0.0000214 & 2.138 & 4496600000 & 1.94098 & 1940.98 \\
\hline \multicolumn{7}{|l|}{ exopanets } \\
\hline HD 149026 b & 102813 & 0.0000435 & 4.35 & 5984000 & 0.00258 & 2.58 \\
\hline HD 209458 b & 192775 & 0.0000831 & 8.31 & 6732000 & 0.00291 & 2.91 \\
\hline TrES-3 & 191346 & 0.0000825 & 8.25 & 2992000 & 0.00129 & 1.29 \\
\hline HD 17156 b & 137084 & 0.0000591 & 5.91 & 23846240 & 0.01029 & 10.29 \\
\hline $\mathrm{xO}-3 \mathrm{~b}$ & 171355 & 0.0000759 & 7.59 & 6791840 & 0.00293 & 2.93 \\
\hline
\end{tabular}

Table 1: The data used in the model for the diameters (2,3,4 columns) and stellar distances (5,6,7 columns) of planets and exoplanets. In the upper part of the table the planets of the Solar System, below it exoplanets are listed. 
Citation: Kereszturi A (2013) How to Size an Exoplanet? A Model Approach for Visualization. Astrol Outreach 1: 101. doi:10.4172/23322519.10001012519.1000101

mass relatively to Jupiter's, the later was taken to be equal to $1 \mathrm{~kg}$. The rocks were put right below the corresponding exoplanet model globe and the students could lift them (after testing the $1 \mathrm{~kg}$ Jupiter model) when the lecturer mentioned how large is the weight of that certain hot Jupiter (Figure 4).

To quantify how effective was the visualization using the exoplanet

$$
\text { HD } 149026 \text { b }
$$

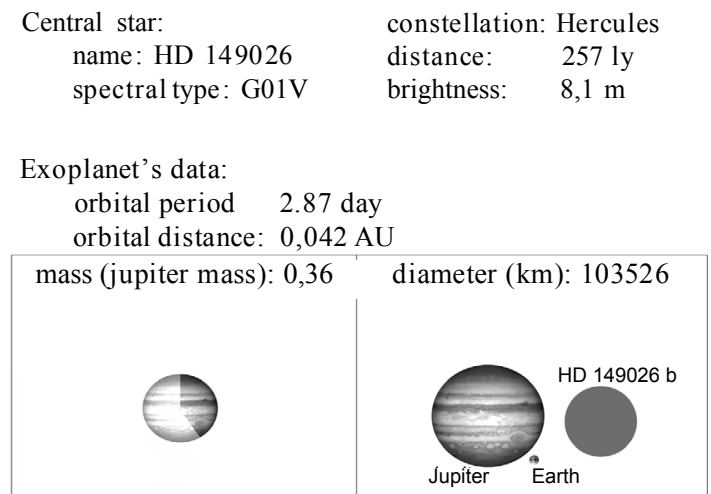

Figure 1: Example sheet of an exoplanet, where the parameters are written in Hungarian language. The mass (lower left), and size (lower right) are visualized graphically below.

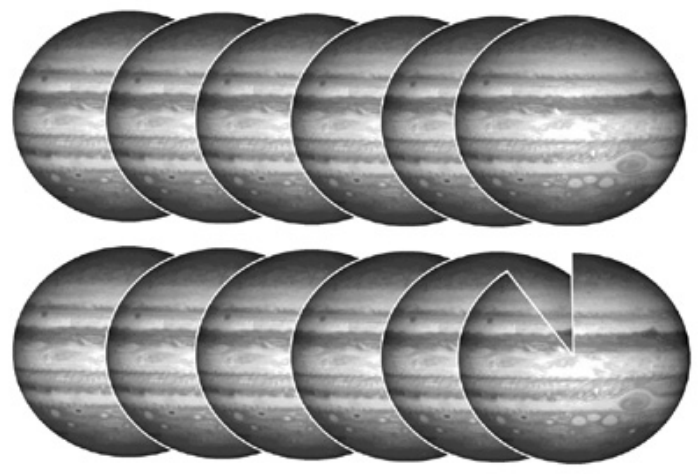

Figure 2: Visualizing 11.8 Jupiter mass.
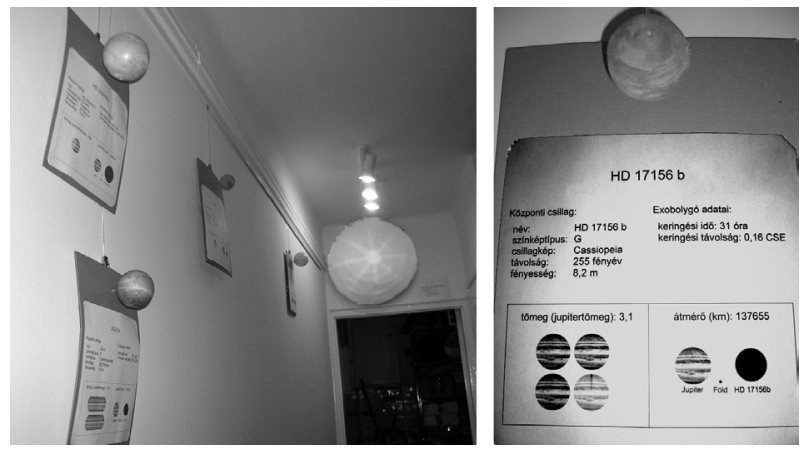

Figure 3: Exoplanet plates hanging on the wall (left) with the solar globe in the background below the ceiling for the Sun. One exopanet's plate is visible on the right, with the plastic planet model at the top and text plus graphical representation at the bottom.

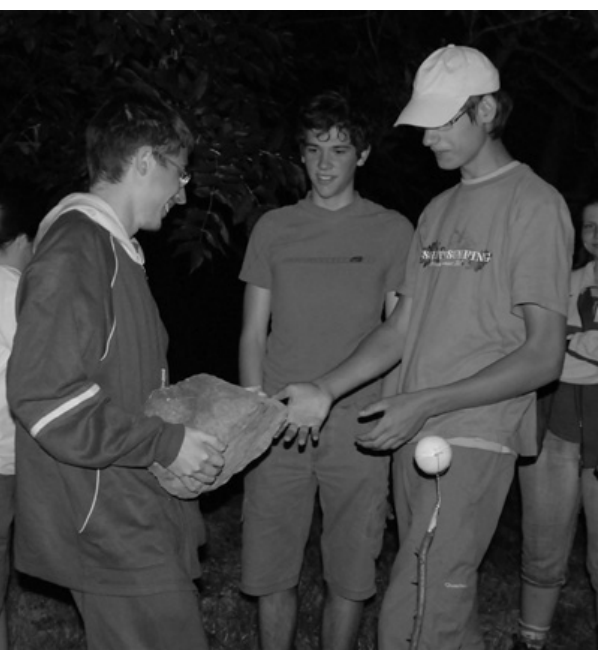

Figure 4: The globe (bottom right) to visualize the size of $\mathrm{XO} 3 \mathrm{~b}$ exoplanet and a rock (on the left in hand) to "visualize" its 11.8 Jupiter mass.

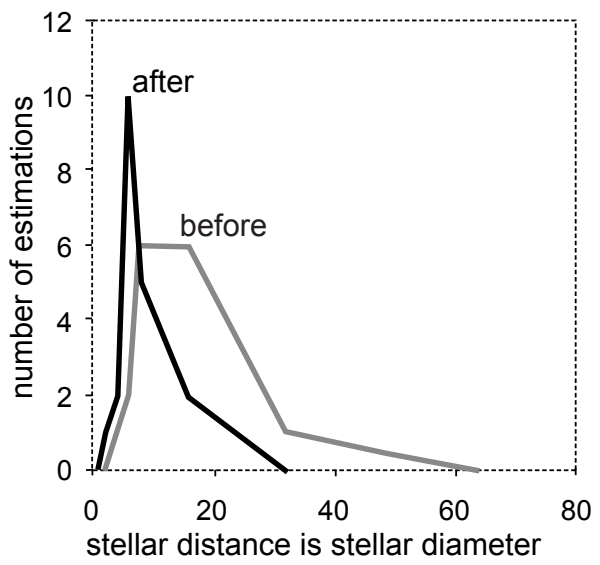

Figure 5: Distribution of the estimations on the typical distance of a hot Jupiter from its central star in the unit of the planet's diameter (equal to Jupiter's), before (gray) and after (black) the presentation.

model, we made the audience to estimate the roughly typical distance of a hot Jupiter (like those indicated in the table) from its central star, in the unit of the diameter of central star. We made this written survey before and after the lecture. The result is visible in Figure 5. It clearly demonstrates the visualization was effective, and the scatter of estimations substantially decreased after the lecture. It is possible that the oral discussion also contributed to decrease the range where the estimated values scattered. But the lecturer did not mention numerical ratios or other values that should be estimated, and did not even call the attention what to look for or memorize fom the model. Although the oral discussion could play some role, alone (without a model) would not have been enough to improve the numerical values as much as it is visible.

\section{Discussion}

Using the experiences, partly from written tests, and partly from our personal feelings during the discussions with the audience we gained the following conclusions. The most important findings might help for 
other educators to improve and adapt such models to other conditions. The experiences are listed in two groups, the first concerns with the physical realization of the model, the second with the feedbacks from the audience.

\section{Experiences from the model realization}

- Using only one central star model with a size equal to the Sun is not only practical but also reasonable as most exoplanet research projects aim the identification of Earth like objects in the habitable zone of a Sun like star.

- Exoplanet and solar system planet visualization together can be realized without restrictions (keeping both the distance and size scales proportional) only in the outdoor, for example sport fields or passage of a school. While in the classroom it is still useful to indicate only Mercury beside the exoplanets, as the distance of Mercury gives useful background for comparison.

- Beside the physical models (globes with given diameters) short description and simple visualizing graphics could be fused effectively. Here the best method is to use the same sized and styled graphics (for example sizes and masses of planets) as it helps the student or visitor to compare them.

\section{Experiences from the usage of the model}

- The students enjoyed the discussion on the basic parameters of exoplanets together with the use of the model both in the observatory and in the astronomy camp too. Simple values like a large mass or small orbital distance could serve as a topic of a conversation.

- The sizes of the hot Jupiter's and their distances could be far better understood with this model than simply memorizing the corresponding numerical values.

- The feedbacks from the audience straightened that this model expanded their view how a planetary system may look like. A planetary system might be substantially more crowded close to its central star than is the Solar System we are living in. This observation opened the view on range of possible planetary systems in their mind. Such a change in imagination might help for the students to apply known (observing, modeling, measuring) methods for planetary systems differ from our one.

- A simple poll was conducted before and after the lecture on the students' view: how they imagine their future, do they want to work on exoplanet research. Before the lecture between 15 and $25 \%$ of student indicated they would like to work on this topic as a researcher (adults were excluded from the survey). After the model demonstration this ratio increased only a little (by $1-4 \%$ ), so such a simple demonstration naturally might not be enough to orient them toward a job close to astronomy or astrobiology.

- It is worth mentioning that beyond these points; the feedbacks from many of the participants in the audience suggest the models helped to have a better image in their mind even if they already had some rough knowledge on the sizes and orbital distances of hot Jupiter's. Based on the personal discussions, the visualization improved the view of these exoplanets in the mind of even those persons who used to read astronomy papers regularly, as the hands-on experience completed well the numerical values.

It is important to note that the dominance of hot Jupiter's among the known exoplanets was characteristic only for the first period of the exoplanet research era. It is known that because of the used Doppler method [15-17] the selection effect enhanced the discovery of larger objects closer to their star, producing dominance of hot Jupiter's. Without this selection effect, using improved methods, it turned out that smaller objects are more numerous than hot Jupiter's [18] and there are many multiple planetary systems [19]. This selection effect should be emphasized in the future during the presentation of similar exoplanet models. In the realization of these works new catalogues (including habitability related issues at the website of the University of Puerto Rico at Arecibo [20], and the extra solar Planets Encyclopedia [21]) help to select representative example exoplanets among the discovered ones.

\section{Conclusions}

The installation of Solar System and exoplanet models at the same model set is useful, as 1. It provides a simple way for visualization Solar System and exoplanet related scales with the same model set, 2 . it gives far better possibility for comparison, 3 . the model could be used in the classroom with some restrictions (indicating only Mercury from the Solar System with keeping distance and size scales a the same time, while the rest of the Solar System might be indicated out of scale, emphasizing that only Mercury was proportional in the model).

The model above all helps to imagine better the real sizes and orbital distance of exoplanets (here hot Jupiter's), especially relative to the Solar System objects. Beyond providing a more accurate image on the basic parameters of the exoplanets, the model opened wider the possible range of parameters how one imagines a planetary system. Earlier mental views were more shaped according to only our knowledge on the Solar System, but after the visualization using this mode the students change their view.

This exoplanet model worked for the years around 2000. Using the more recent discoveries, the growing number of Neptune and super Earth sized planets, require a further developed version of models, but the methods used here are still valid and might help the work of the visualization in the classroom and for the public.

\section{Acknowledgment}

This work was supported by the educational issues of the OTKA PD-105970 and K-104607 projects. The author thanks to Anna Reith and Agnes Szabo for their help in the realization of the exoplanet models. The suggestions of the two referees are also acknowledged.

\section{References}

1. Kereszturi A, Hyder D (2012) Planetary Science in Higher Education: Ideas and Experiences. Journal of Geography in Higher Education 36: 499-525.

2. Kereszturi A, Pentek K (2012) New Planetary Science Course at the University of Western Hungary. 43rd Lunar and Planetary Science Conference, abstract 1778.

3. Kereszturi A (2010) Visualization in the Education of Astrobiology. In Astrobiology: Physical Origin, Biological Evolution and Spatial Distribution, ed. Simon Hegedűs and Jakob Csonka, Nova Publishers 131-141.

4. Kereszturi A (2009) Online astrobiology course in Hungary. 40th Lunar and Planetary Science Conference, abstract 1048.

5. D'Cruz, Noella L (2012) Astr 101 Students Attitudes Towards Essays On Transits, Eclipses and Occultations. American Astronomical Society, AAS Meeting abstract 220: 437.08.

6. Gould RR, Sunbury S, Sienkiewicz F, Deutsch F, Ibrahim A, et al. (2011) The Other Worlds Project: Students Detect Exoplanets Using the MicroObservatory Online Telescopes. Earth and Space Science: Making Connections in Education and Public Outreach, Proceedings of the Astronomical Society of the Pacific conference, Ed. Jensen JB, Manning JG, Gibbs MG. San Francisco 443: 162. 
Citation: Kereszturi A (2013) How to Size an Exoplanet? A Model Approach for Visualization. Astrol Outreach 1: 101. doi:10.4172/23322519.10001012519.1000101

Page 5 of 5

7. Gould A, Koch D, Devore E, Harman P (2008) Preparing for the 2009 International Year of Astronomy: A Hands-On Symposium ASP Conference Series 400, Ed.: Gibbs MG, Barnes J, Manning JG, Partridge B. San Francisco: Astronomical Society of the Pacific 400: 230 .

8. Stevens L, Carson J, Ruwadi D, Low K, Jordan S, et al. (2013) The Hubble Exoplanet Classroom. American Astronomical Society, AAS Meeting 221: 246.05 .

9. Gould RR, Sunbury S, Krumhansl R (2012) Using online telescopes to explore exoplanets from the physics classroom. American Journal of Physics 80: 445451.

10. Dartnell LR, Burchell MJ (2009) Survey on Astrobiology Research and Teaching Activities within the United Kingdom. Astrobiology 9: 717-730.

11. Wisniewski, John P, Larson A (2010) Exoplanet Peer-Learning Exercises for Introductory Astronomy Courses. American Astronomical Society, AAS Meeting; Bulletin of the American Astronomical Society 42: 507.

12. Kitchin CR (2012) Exoplanets. Finding, Exploring, and Understanding Alien Worlds, Springer.

13. Seager S (2012) Exoplanets. University of Arizona Press.
14. Casoli F, Encrenaz T (2007) The New Worlds: Extrasolar Planets. Springer.

15. Marcy G, Butler RP, Fischer D, Vogt S, Wright JT, et al. (2005) Observed Properties of Exoplanets: Masses, Orbits, and Metallicities. Progress on Theoretical Physics Supplement 158: 24-42.

16. Lineweaver $\mathrm{CH}$, Grether D (2003) What Fraction of Sun-like Stars Have Planets? The Astrophysical Journal 598: 1350-1360.

17. O'Toole S, Tinney C, Jones H (2008) Selection effects in Doppler velocity planet searches. Exoplanets: Detection, Formation and Dynamics, Proceedings of the International Astronomical Union, IAU Symposium 249: 111-114.

18. Fressin F, Torres G, Rowe JF, Charbonneau D, Rogers LA, et al. (2012) Two Earth-sized planets orbiting Kepler-20. Nature 482: 195-198.

19. Lyons R, Ho S, Turner EL, Kokubo E (2013) Statistical Analysis of Multiplanetary Systems. American Astronomical Society, AAS Meeting 221: 435.06.

20. The Habitable Exoplanets Catalog. Planetary Habitable Laboratory, University of Puerto Rico at Arecibo.

21. The Extrasolar Planets Encyclopedia. Expoplanet.eu. 